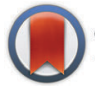

CrossMark \& click for updates

Cite this: J. Mater. Chem. B, 2016 , 4, 3685

Received 26th November 2015, Accepted 16th April 2016

DOI: $10.1039 / c 5 t b 02489 g$

www.rsc.org/MaterialsB

\title{
Fabrication of living soft matter by symbiotic growth of unicellular microorganisms $\dagger$
}

\author{
Anupam A. K. Das, ${ }^{a}$ James Bovill, ${ }^{a}$ Maram Ayesh, ${ }^{a}$ Simeon D. Stoyanov ${ }^{b c}$ and \\ Vesselin N. Paunov*a
}

\begin{abstract}
We report the fabrication of living soft matter made as a result of the symbiotic relationship of two unicellular microorganisms. The material is composed of bacterial cellulose produced in situ by acetobacter (Acetobacter aceti NCIMB 8132) in the presence of photosynthetic microalgae (Chlamydomonas reinhardtii cc-124), which integrates into a symbiotic consortium and gets embedded in the produced cellulose composite. The same concept of growing living materials can be applied to other symbiotic microorganism pairs similar to the combination of algae and fungi in lichens, which is widespread in Nature. We demonstrate the in situ growth and immobilisation of the C. reinhardtii cells in the bacterial cellulose matrix produced by the simultaneous growth of acetobacter. The effect of the growth media composition on the produced living materials was investigated. The microstructure and the morphology of the produced living biomaterials were dependent on the shape of the growth culture container and media stirring conditions, which control the access to oxygen. As the photosynthetic C. reinhardtii cells remain viable and produce oxygen as they spontaneously integrate into the matrix of the bacterial cellulose generated by the acetobacter, such living materials have the potential for various applications in bio-hydrogen generation from the immobilised microalgae. The proposed approach for building living soft matter can provide new ways of immobilising other commercially important microorganisms in a bacterial cellulose matrix as a result of symbiosis with acetobacter without the use of synthetic binding agents and in turn increase their production efficiency.
\end{abstract}

\section{Introduction}

Symbiosis is the relationship between two or more organisms involved to create conditions favourable for mutual growth and proliferation of each other with important implications in the ecology of the system. ${ }^{1}$ Lichens are one of the most well-studied examples of symbiosis to exist naturally in the environment. ${ }^{2-4}$ They consist mainly of a filamentous fungus (mycobiont) and a photosynthesising alga or a cyanobacterium (photobiont). ${ }^{5,6}$ The interactions between the two organisms are not random and depend on various environmental parameters. Lichens can survive in harsher environments compared to the constituting fungi and algae as individual living microorganisms thanks to passing nutrients and metabolites to each other which ensures better adaptability of the lichen. ${ }^{7}$ In this study, we are aiming to

\footnotetext{
${ }^{a}$ Department of Chemistry, University of Hull, Hull, UK.

E-mail:v.n.paunov@hull.ac.uk; Tel: +44 (0)1482 465660

${ }^{b}$ Laboratory of Physical Chemistry and Soft Matter, Wageningen University, 6703 HB Wageningen, The Netherlands

${ }^{c}$ Department of Mechanical Engineering, University College London, Torrington Place, London WC1E JJE, UK

$\dagger$ Electronic supplementary information (ESI) available. See DOI: 10.1039/ c5tb02489g
}

develop a new concept for the preparation of living soft matter by mimicking the relationship between two different microorganisms living symbiotically. Two different classes of microorganisms were grown together in a specific medium where one of the cultures produces the substrate for the growth and proliferation of the other culture, which in turn uses the metabolites produced by the first microorganism and produces other common metabolites. There is evidence that bacterial species can be associated with microalgal species, ${ }^{8}$ which suggests that there are specific mechanisms of interaction which benefit both the microalgae and the bacteria to enable their coexistence in the same habitat. There are other examples, where Pseudomonas sp., a gram negative bacterium can grow symbiotically along with Chlorella vulgaris. ${ }^{9}$

In this study, we explore the symbiotic combination of the acetobacter (Acetobacter aceti NCIMB 8132) and a photosynthesising microalga (Chlamydomonas reinhardtii cc-124). Our aim is to use their symbiotic relationship to fabricate a new type of living biomaterial (tissue) by growing the microalgae in the same culture media with the acetobacter and immobilise their cells in situ in the bacterial cellulose gel which is one of the by-products of the acetobacter metabolism.

Previous research has shown that many different cultures can be immobilised or grown on already produced bacterial 
cellulose (nata de coco). Other microorganisms have been successfully immobilised on bacterial cellulose for efficient generation of various kinds of by-products. Yeast has been grown on bacterial cellulose to increase the wine production efficiency and improve the process economy. ${ }^{10}$ Cellulose fibres produced by Acetobacter xylinum were used to immobilise Pseudomonas Stutzeri for the process of biological denitrification of waste water for the removal of nitrates and their conversion into nitrogen gas. ${ }^{11}$ The bacterial cellulose made by Glucanobacter xylinus was shown to be highly biocompatible by growing human osteoblast cells with rapid attachment and extension. ${ }^{12}$ In our case, the immobilisation of the microalgae was done in real time during the production of the bacterial cellulose gel along with a symbiotic relationship with the acetobacter which results in the growth and proliferation of the living biomaterial. It has been previously reported that the microalgae $C$. reinhardtii have the ability to produce biohydrogen in suspension culture as well as immobilised culture with the latter found to be more efficient, ${ }^{13}$ hence the living soft matter may have advantages like improved hydrogen production from immobilised culture and various other metabolite extractions.

Here we immobilised the microalgae cells in real time with the bacterial cellulose produced by the acetobacter in the same culture consortia. We report the production of a new living soft matter using two different unicellular microorganisms microalgae and cellulose producing bacteria which normally do not form colonies as single cultures. The acetobacter cells are aerobes that use sugars with the production of cellulose and acetic acid as their major byproducts. ${ }^{14}$ This bacterial cellulose matrix was simultaneously used as a substrate for the growth of the photosynthesising microalgae $C$. reinhardtii. The microalgae in turn provide oxygen and pass it onto the cellulose producing bacteria (see Scheme 1) in their environment. This allowed the acetobacter to produce cellulose not only at the air-water interface but also around the photosynthetic microalgae which get integrated in the cellulose gel. The schematics of the metabolite exchange during the symbiotic growth is shown in Fig. 1.

The paper is organised as follows. We study the optimal composition of the media which favours symbiotic growth of the living soft matter. The viability of the integrated microalgae and the microstructure of the produced living materials under different growth media conditions is also examined. We monitor the media acidity during the symbiotic growth of the living materials at different initial ratios of the two cultures. We discuss the similarities with lichens in Nature and the possible application of this new approach for growing other types of living materials.

\section{Materials and methods}

\section{Materials}

Microalgae cultures were grown in the tris-acetate-phosphate (TAP) culture medium at an incubation temperature of $30{ }^{\circ} \mathrm{C}$. The culture medium of $C$. reinhardtii consisted of TAP salts
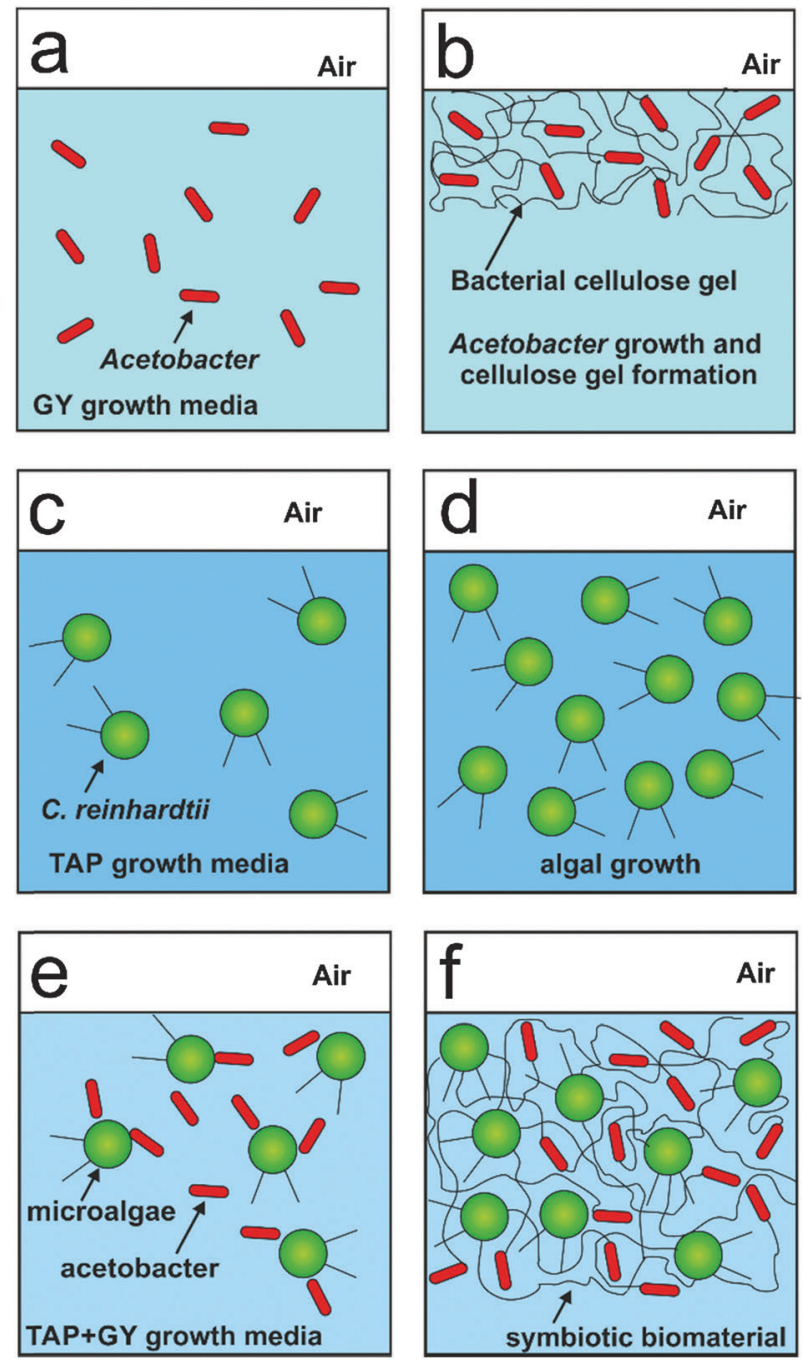

Scheme 1 Scheme of ( $a$ and $b$ ) the growth of acetobacter in GY media with the formation of bacterial cellulose gel; (c and d) the growth of the microalgae in TAP media. (e and f) The growth of a mixed culture of acetobacter and microalgae in mixed media, TAP + GY media which allows the two microbial cells to form a symbiotic living biomaterial.

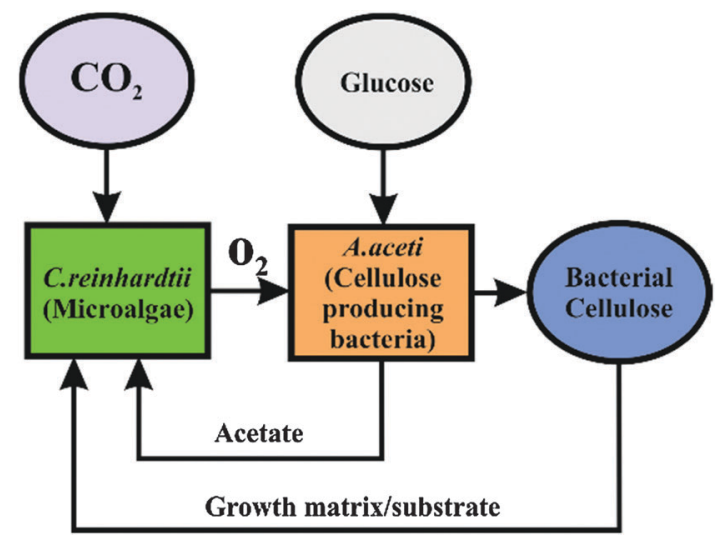

Fig. 1 Schematics showing the symbiosis between the microalgae (C. reinhardtii) and the cellulose producing bacteria (A. aceti) with the passing of nutrients in between the two unicellular microorganisms. 
(ammonium chloride, $\mathrm{NH}_{4} \mathrm{Cl}$; magnesium sulphate, $\mathrm{MgSO}_{4} \cdot 7 \mathrm{H}_{2} \mathrm{O}$ and calcium chloride $\mathrm{CaCl}_{2} \cdot 2 \mathrm{H}_{2} \mathrm{O}$ ), phosphate buffer solution and Hutner's trace element solution (EDTA disodium salt, $\mathrm{ZnSO}_{4} \cdot 7 \mathrm{H}_{2} \mathrm{O}, \mathrm{H}_{3} \mathrm{BO}_{3}, \mathrm{MnCl}_{2} \cdot 4 \mathrm{H}_{2} \mathrm{O}, \mathrm{CoCl}_{2} \cdot 6 \mathrm{H}_{2} \mathrm{O}, \mathrm{CuSO}_{4} \cdot 5 \mathrm{H}_{2} \mathrm{O}$, $\mathrm{FeSO}_{4} \cdot 7 \mathrm{H}_{2} \mathrm{O},\left(\mathrm{NH}_{4}\right)_{6} \mathrm{Mo}_{7} \mathrm{O}_{24} \cdot 4 \mathrm{H}_{2} \mathrm{O}$, all purchased from SigmaAldrich, UK). Acetobacter cultures were grown in glucose/yeast extract (GY) media at an incubation temperature of $28{ }^{\circ} \mathrm{C}$. The acetobacter growth media consist of glucose purchased from Fisher Scientific, UK and yeast extract purchased from Oxoid Ltd, UK. The fluorescein diacetate (FDA) assay used to determine the viability of the $C$. reinhardtii cell was purchased from Sigma Aldrich, UK. Deionised water produced using a Milli-Q reverse osmosis system (Millipore, UK) was used in all experiments.

\section{Algal strain and growth conditions}

The $C$. reinhardtii cc- 124 strain was kindly provided by the research group of Prof Michael Flickinger at North Carolina State University, USA. The cells were grown photoheterotrophically in acetate-rich medium in 100, 250 and $500 \mathrm{~mL}$ Erlenmeyer flasks containing 50, 150 and $250 \mathrm{~mL}$ of standard tris-acetate-phosphate (TAP + S) medium at $\mathrm{pH} 7.0 .^{15}$ The flask was placed in a water jacket connected to a thermostatic water bath maintained at $25-30{ }^{\circ} \mathrm{C}$ and placed on a magnetic stirrer with a stirring bar to keep the culture agitated at $500 \mathrm{rpm}$. The setup was illuminated using two $23 \mathrm{~W}$ cool white lights (2800 Lux) providing photosynthetically active radiation of $36.4 \mu \mathrm{E} \mathrm{m}^{-2} \mathrm{~s}^{-1}$. The cells were grown up to three days and harvested before their late logarithmic growth phase by centrifugation at $3000 \mathrm{rpm}$ for $5 \mathrm{~min}$. All glassware was autoclaved at $121{ }^{\circ} \mathrm{C}$ for $20 \mathrm{~min}$ before use for cell growth.

\section{Acetobacter strain and growth conditions}

The $A$. aceti (NCIMB 8132) was purchased from NCIMB Ltd, UK. The cells were grown in glucose and yeast extract media (GY media) in $250 \mathrm{~mL}$ Erlenmeyer flasks containing $150 \mathrm{~mL}$ of GY media. ${ }^{16}$ The flask was placed in a Stuart orbital incubator SI500 maintained at $28{ }^{\circ} \mathrm{C}$. The cells were grown for up to seven days along with the formation of different layers of cellulose produced by the acetobacter.

\section{Microalgae immobilisation procedure and symbiosis}

The microalgae (C. reinhardtii) were immobilised in the bacterial cellulose gel produced by $A$. aceti. The cells were grown together in a mixture of TAP and GY media at various concentrations of $100 \%$ GY media (only A. aceti), 100\% TAP media (only C. reinhardtii), $100 \% \mathrm{GY}$ media (with $C$. reinhardtii and $A$. aceti), 90:10 GY:TAP media (v/v), 80:20 GY:TAP media (v/v), and 70:30 GY:TAP media (v/v). The initial inoculum amounts and their ratio were also varied for both microorganisms in the mixture as shown in Tables 1 and 2. The microalgae cells integrated into the bacterial cellulose gel composite were formed in different culture containers, such as a beaker, conical flask, Petri dish and leaf-shaped container, in order to mould the living soft matter into various sizes and shapes. The $\mathrm{pH}$ of the culture media was measured at various times during
Table 1 The table showing the samples grown in $70: 30 \mathrm{GY}$ : TAP media $(\mathrm{v} / \mathrm{v})$ type containing different amounts of $C$. reinhardtii (grams of wet weight) and $A$. aceti ( $\mathrm{mL}$ of broth) used for reference to the graphs shown in Fig. 4

\begin{tabular}{lll}
\hline Sample & A. aceti $(\mathrm{mL})$ & C. reinhardtii $(\mathrm{g})$ \\
\hline 1 & 2 & 0.35 \\
2 & 4 & 0.35 \\
3 & 6 & 0.35 \\
4 & 12 & 0.35 \\
5 & - & 0.5 \\
6 & 10 & -
\end{tabular}

Table 2 The table showing samples grown in the $70: 30 \mathrm{GY}$ : TAP media $(\mathrm{v} / \mathrm{v})$ type containing different amounts of $C$. reinhardtii (grams of wet weight) and $A$. aceti ( $\mathrm{mL}$ of broth) used for reference to the graphs shown in Fig. 5

\begin{tabular}{lll}
\hline Sample & A. aceti $(\mathrm{mL})$ & C. reinhardtii $(\mathrm{g})$ \\
\hline 7 & 10 & 0.35 \\
8 & 10 & 0.45 \\
9 & 10 & 0.55 \\
10 & 10 & 0.60 \\
5 & - & 0.5 \\
6 & 10 & -
\end{tabular}

the symbiotic growth using a Fisher brand Hydrus $300 \mathrm{pH}$ meter connected to a Jenway electrode.

\section{Microscopy studies of the living soft matter}

The viability of $C$. reinhardtii cells was monitored using a BX-51 fluorescence microscope equipped with a DP70 digital camera and fluorescein diacetate (FDA) as a live/dead cell assay. Fluorescein diacetate (FDA) is a non-fluorescent compound which easily diffuses into intact cells. ${ }^{17,18}$

The result is the uptake and hydrolysis of the non-fluorescent precursor (FDA) by esterase inside the cell and accumulation of a fluorescent byproduct (fluorescein). ${ }^{19}$ We used $0.5 \%$ (w/v) FDA dissolved in acetone. $20 \mu \mathrm{L}$ of this solution was used per $\mathrm{mL}$ of the cell sample. The tube with the mixture was shaken for 10 minutes using a vortex at $1500 \mathrm{rpm}$ under dark conditions to avoid the photobleaching of the produced fluorescein as a result of the enzymatic hydrolysis of FDA on the inside of the viable cells. The sample was washed three times with Milli-Q water using a centrifuge (Eppendorf Minispin plus) at $3500 \mathrm{rpm}$ for 5 minutes in order to remove any extracellular fluorescein. The SEM images were obtained using a Zeiss Evo 60 Scanning Electron Microscope. The living soft matter samples were fixed using $2.5 \%$ glutaraldehyde in phosphate buffer followed by incubation in $1 \%$ osmium tetroxide in phosphate buffer for 1 hour. They were then gradually dehydrated in ethanol (30\%, 50\%, 70\%, 90\% and two times dry $100 \%$ ) before being dried (at the critical point) using liquid carbon dioxide in a critical point dryer B7010. The samples were coated with $\mathrm{Au} / \mathrm{Pd}$ after fixing using a Polaron Sputter Coater, model SC7640 fitted with a coating thickness monitor.

They were mounted on aluminium SEM stubs and evacuated inside the coater using a rotary vacuum pump. Vacuum was regulated with a leak valve connected to an argon supply 


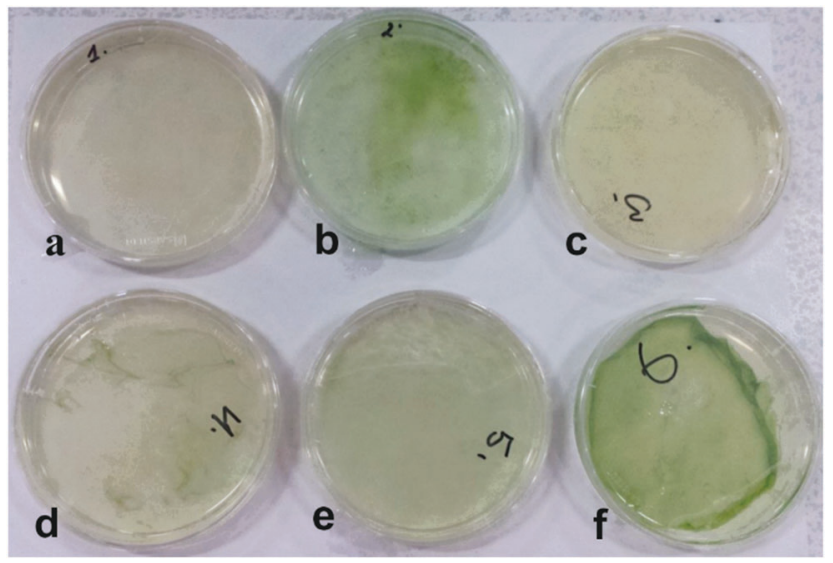

Fig. 2 Samples of living soft matter produced by C. reinhardtii (microalgae) and $A$. aceti (cellulose producing bacteria) grown together in a Petri dish containing different concentrations of glucose-yeast (GY) and trisacetate-phosphate (TAP) culture media. (a) 100\% GY media (only A. aceti) (b) 100\% TAP media (only C. reinhardtii), (c) 100\% GY media (with C. reinhardtii and A. aceti), (d) $90: 10 \mathrm{GY}:$ TAP media (v/v), (e) $80: 20 \mathrm{GY}:$ TAP media (v/v), and (f) $70: 30 \mathrm{GY}$ : TAP media (v/v).

system. The operating pressure was 2-4 Pa. A direct current voltage (about $1 \mathrm{kV}$ ) was applied to the source material (Au/Pd target), which ionises the argon gas. Argon ions were accelerated towards the target, ejecting metal atoms in the process which coat the samples. The samples were rotated at about $0.5 \mathrm{rpm}$ during sputtering. The sputtering process was terminated automatically by the thickness monitor at a coating thickness of approximately $2-3 \mathrm{~nm}$.

\section{Mechanical property testing}

The mechanical tensile stress properties of the produced cellulose and biomaterials were measured using a tensile machine at a stretch rate of $0.4 \mathrm{~mm} \mathrm{~s}^{-1}$. The samples were cut to make uniform $2 \times 1 \mathrm{~mm}$ pieces for testing. Samples were fixed between the upper and the lower clamps in order to prevent slip with force applied to the upper clamp to pull the sample in tension. The tensile experiments were performed at room temperature. The tensile stress $\sigma^{*}$ measured in Pa is defined as the force applied on the cellulose material $(F)$ divided by the measured crosssectional area of the sample $(A)$ as shown in eqn (1)

$$
\sigma^{*}=F / A
$$

The tensile strain $(\varepsilon)$ was calculated using eqn (2):

$$
\varepsilon=\Delta L / L_{0}=\left(L-L_{0}\right) / L_{0}
$$

The tensile strain is defined as the ratio of change in the length $(\Delta L)$ to the initial length of the cellulose. The elastic or Young's modulus of the cellulose $(E)$ was calculated using eqn (3):

$$
E=\mathrm{d} \sigma^{*} / \mathrm{d} \varepsilon
$$

The largest tensile stress and strain were calculated from the breaking of the cellulose biomaterial.

\section{Chemical analysis}

Elemental composition $(\mathrm{C}, \mathrm{H}, \mathrm{N})$ of the produced bacterial cellulose (BC) was determined by elemental analysis in a $\mathrm{CHN}$ Carlo Erba EA1108 CHN analyser. Films of BC microfibers were dried overnight at $70{ }^{\circ} \mathrm{C}$ to remove moisture before analysis.
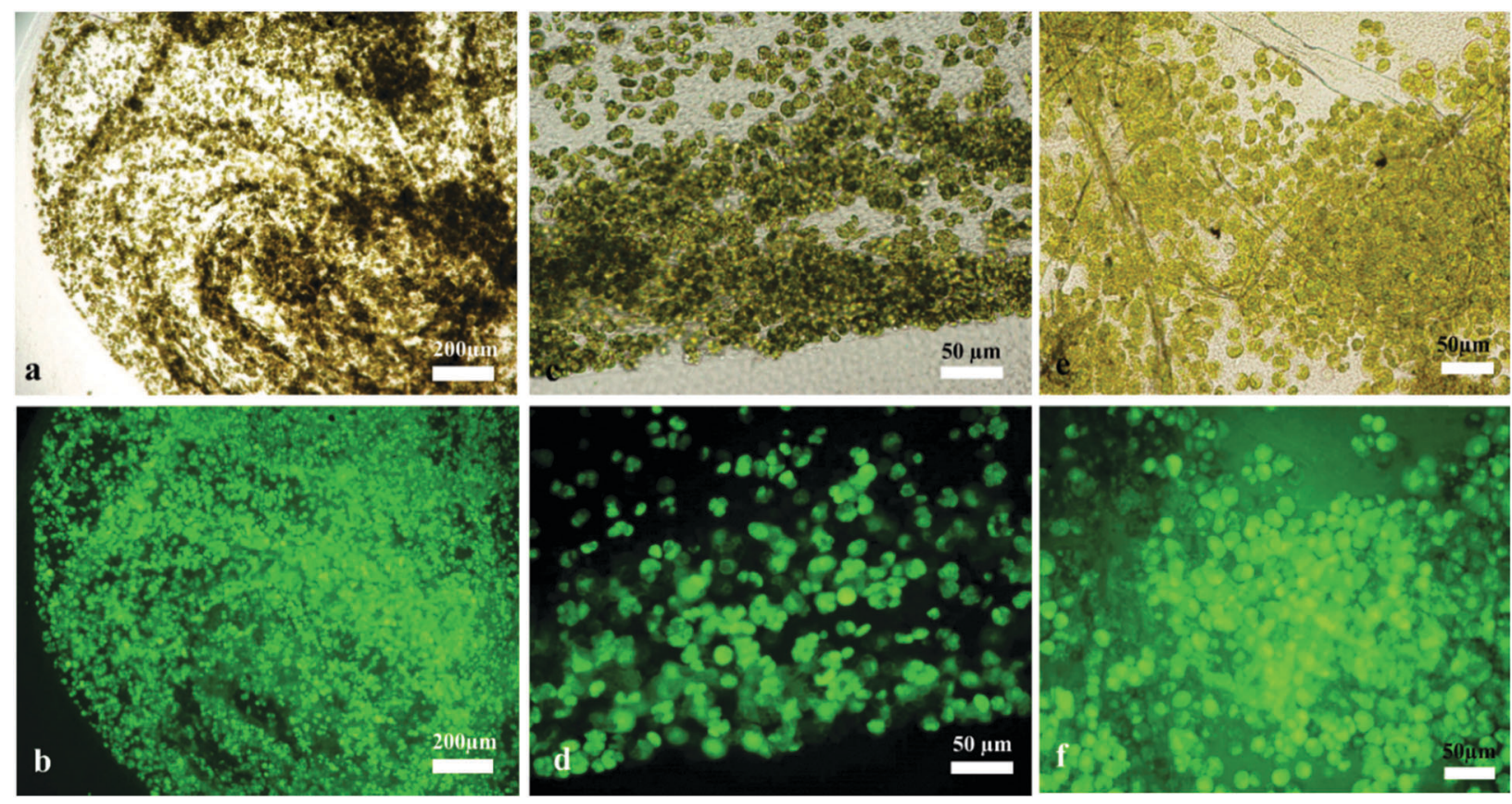

Fig. 3 Optical and fluorescence micrographs showing the viability of $C$. reinhardtii (microalgae) cells immobilised in cellulose produced by $A$. aceti in different growth media concentrations after being treated with FDA, (a) and (b) 100\% GY media, (c) and (d) $70: 30$ GY:TAP media and (e) and (f) $90: 10$ GY: TAP media (v/v). 
The elemental analysis was carried out after washing the cellulose several times with ethanol and deionised water in order to devoid the material of any cells or other media components. The bacterial cellulose and the produced biomaterial were also analysed using a FT-IR spectrophotometer. The samples were dried for 3 hours at $100{ }^{\circ} \mathrm{C}$ to remove moisture before the measurement of the FT-IR spectra. The spectra were recorded in the ATR mode on a Thermo Scientific Nicolet iS5 spectrophotometer in the range between 500 and $4000 \mathrm{~cm}^{-1}$ at a resolution of $4 \mathrm{~cm}^{-1}$ and a total accumulation of 64 scans. The samples were also examined using a Zeiss Evo 60 Scanning Electron Microscope equipped with an EDX energy dispersive X-ray analyser to investigate the elemental composition of the system (Inca energy 350) connected to an $80 \mathrm{~mm}^{2}$ silicon drift detector (SDD).

\section{Results and discussion}

\section{Growth media optimisation}

The cell immobilisation procedure described in the Methods section was used to entrap the microalgae cells within the cellulose gel matrix produced by the acetobacter cells. The cells were grown in mixed media of various ratios of GY and TAP media with acetobacter and microalgae cells as shown in Scheme 1 and Fig. 2. Crucially important for the efficient growth and sustainability of both microorganisms in the system was to determine the optimal ratio of the GY and TAP media which also allows us to immobilise the microalgae efficiently in the cellulose gel produced by the acetobacter. We found that the volume ratio of $70: 30(\mathrm{v} / \mathrm{v}) \mathrm{GY}$ : TAP media is the optimal media formulation for efficient immobilisation of the microalgae within the bacterial cellulose matrix and the simultaneous growth of both microorganisms (see also Fig. 2f). A larger amount of GY media in the system compared to TAP media was needed as more glucose was necessary for the synthesis of bacterial cellulose. The viability of the immobilised $C$. reinhardtii cells in the cellulose gel produced by $A$. aceti was checked using

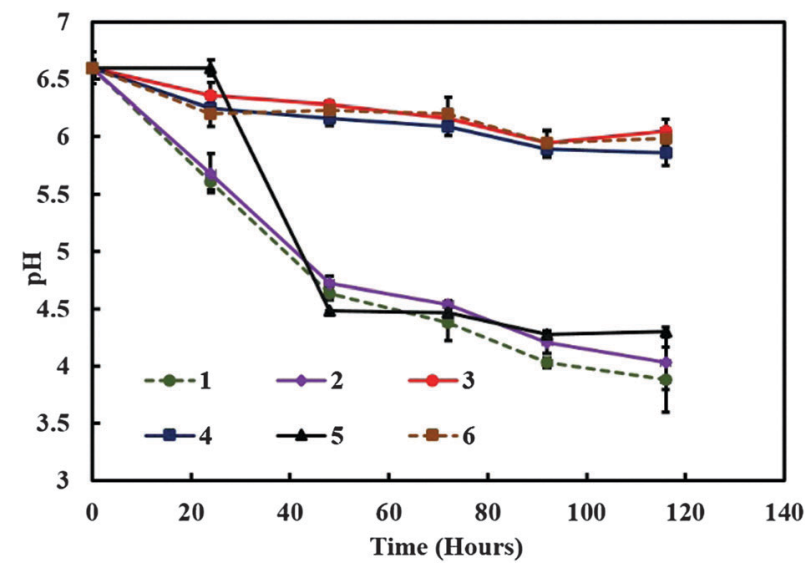

Fig. 4 The media $\mathrm{pH}$ versus time for various mixed cultures containing different amounts of $C$. reinhardtii and $A$. aceti. The numbered lines represent the specific sample setup from Table 1.

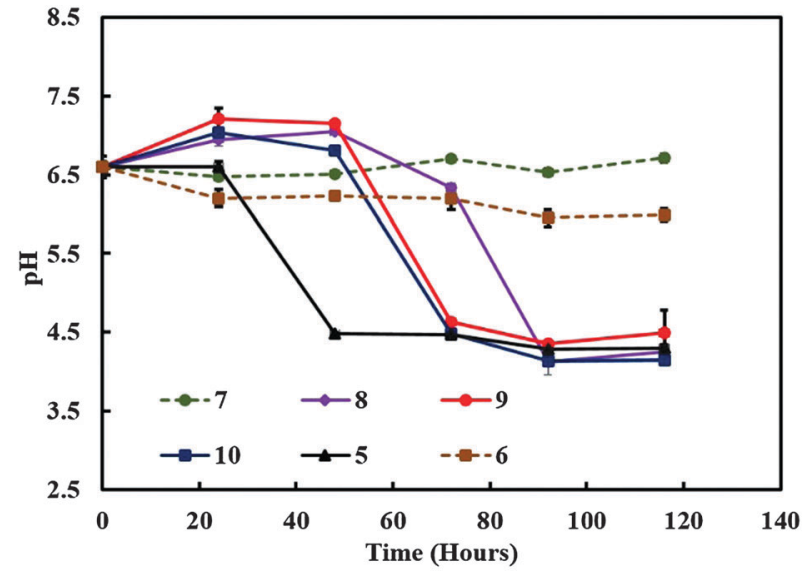

Fig. 5 The media $\mathrm{pH}$ versus time for various mixed cultures containing different amounts of $C$. reinhardtii and $A$. aceti. The numbers represent the specific sample setup from Table 2.

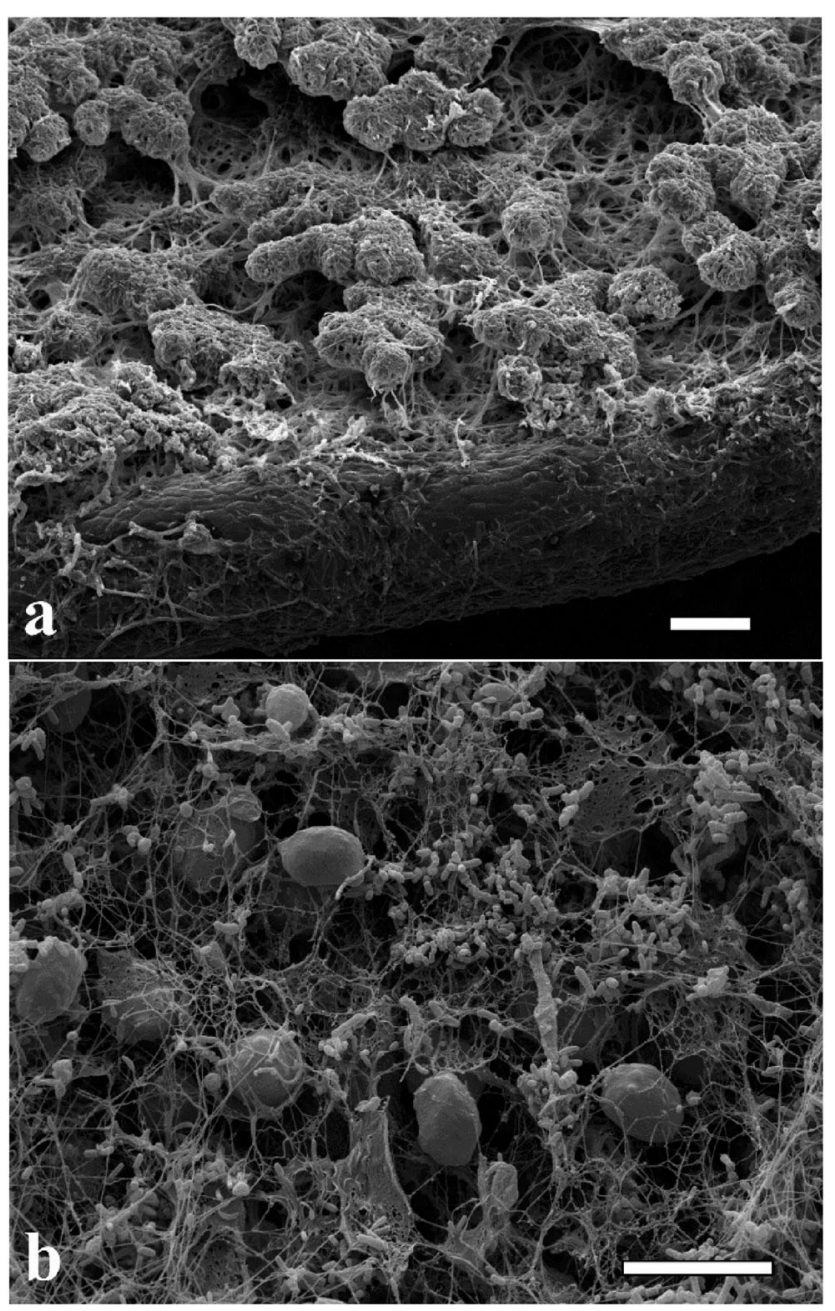

Fig. 6 Scanning electron micrograph of (a) lichen colony growing on a leaf $^{20}$ and (b) the living biomaterial made of cellulose produced from A. aceti and immobilised microalgae C. reinhardtii co-cultured in a glass beaker. Image (a) reproduced with permission from ref. 20 . All scale bars are $10 \mu \mathrm{m}$ in size. 
FDA as a live/dead cell assay at different stages of growth of the living material. The viability of the immobilised cells was maintained for up to 7 days after which the biomaterial was extracted for further analysis. It is evident from Fig. 3 that the immobilised microalgae cells were viable under all media conditions with different ratios of GY and TAP media. However, we found large variations in the morphology and the texture of the produced living biomaterial. More results and analysis including SEM images of these materials are presented in the ESI. $\dagger$

We experimented with various amounts of $A$. aceti and a constant amount of $C$. reinhardtii in the $70: 30 \mathrm{GY}$ : TAP media $(\mathrm{v} / \mathrm{v})$ in the symbiotic culture vessel. The $\mathrm{pH}$ was recorded at various times in order to monitor the change in the culture media acidity which usually varies due to the accumulation of by-products of the cell metabolism. In samples containing both kinds of microorganisms the $\mathrm{pH}$ decreased strongly from the initial value of 6.5 as observed from Fig. 4 . The $\mathrm{pH}$ of the control samples containing only $A$. aceti showed very little change from the initial $\mathrm{pH}$ value with time, while in the case of $C$. reinhardtii it dropped from the initial value of 6.5 to 4.5 as shown in Fig. 4. In another experiment we varied the amount of $C$. reinhardtii cells keeping the amount of $A$. aceti cells constant in the 70:30 GY:TAP media (v/v). It was observed that cultures with a substantial amount of $C$. reinhardtii growth showed a similar decrease in $\mathrm{pH}$ from 6.5 to 4.5 approximately as shown in Fig. 5. Drop in $\mathrm{pH}$ of the media was not observed in the case of cultures without microalgae growth. It can be concluded that under these conditions, the metabolism of the microalgae in the system increases the acidity of the surrounding culture medium. It is known that the microalgae can use the residual glucose present in the culture media in the process of glycolysis in order to produce additional energy (ATP) and release pyruvate (pyruvic acid) as a by-product. ${ }^{20}$ This explains the reduction in the $\mathrm{pH}$ with time as evident from Fig. 4 and 5 with the growth of the microalgae. The drop in the $\mathrm{pH}$ was highly significant and much quicker in the case of culture containing only $C$. reinhardtii compared to cultures containing both the microorganisms, which is due to the availability of ample glucose for the microalgae. Whereas the presence of acetobacter results in the use of glucose for its own metabolism.

These sets of experiments enabled us to pinpoint the specific amount to acetobacter and microalgae required in order to maintain successful symbiotic consortia and in turn control the production of the living biomaterial.

\section{SEM studies of the living soft matter microstructure}

The microalgae $C$. reinhardtii mimic the photosynthetic alga and $A$. aceti mimics the filamentous fungi, which is the other part of the symbiotic pair in lichens. There are also some visual similarities in the morphology between lichens and the produced living biomaterials as presented in Fig. 6. The SEM image shown in Fig. 6a shows a natural lichen ${ }^{20}$ compared to the living biomaterial made in our laboratory as shown in Fig. 6b. The similarity in the microstructure of the immobilised microalgae in lichens compared to our living biomaterial is quite intriguing, although the latter is based on a completely different symbiotic pair and the extracellular matrix of bacterial cellulose nanofibers generated by $A$. aceti.
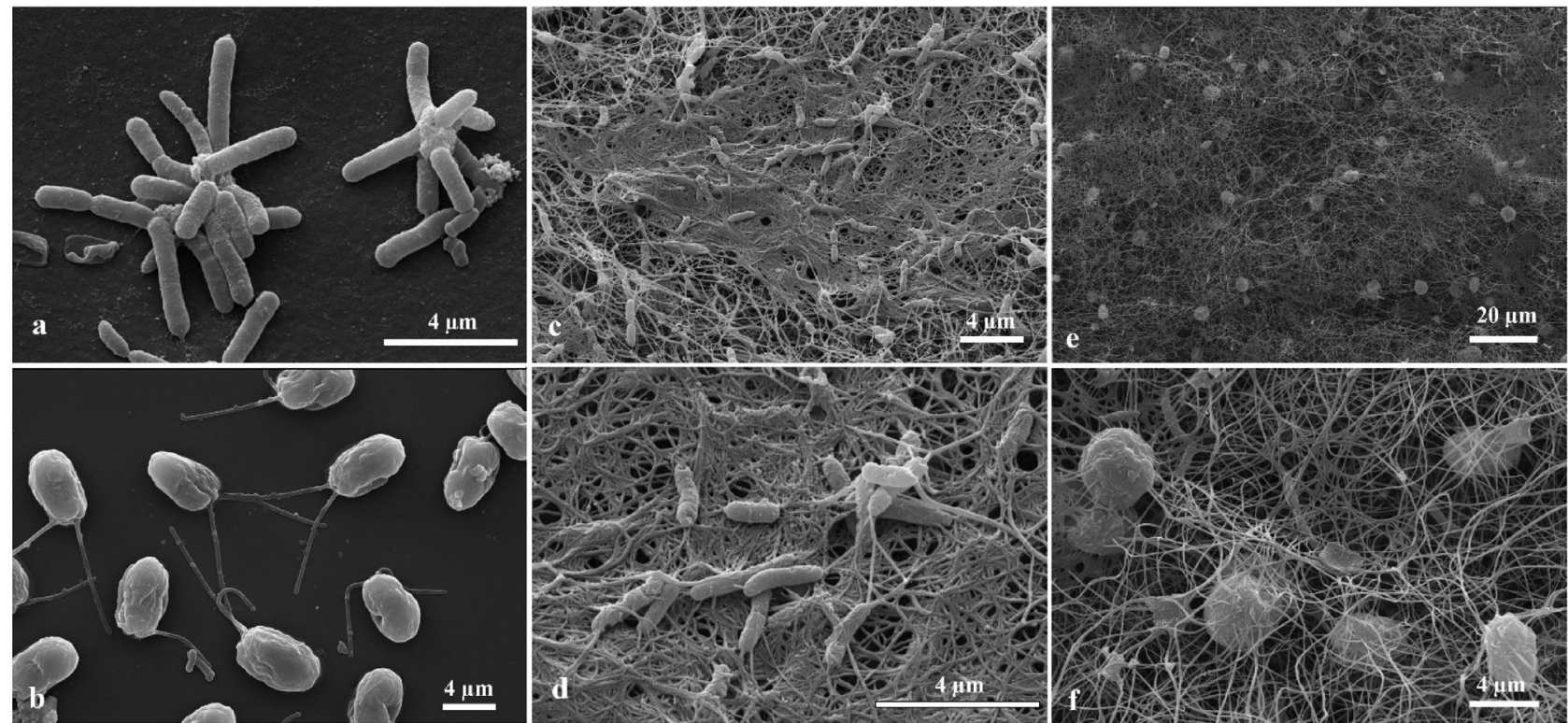

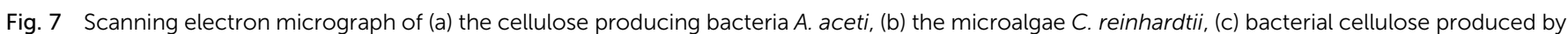

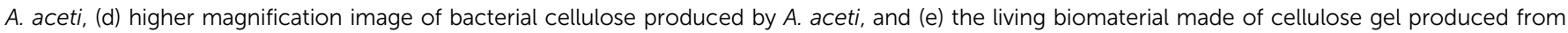

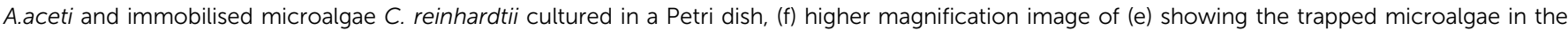

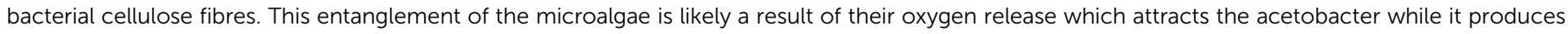
the cellulose. 

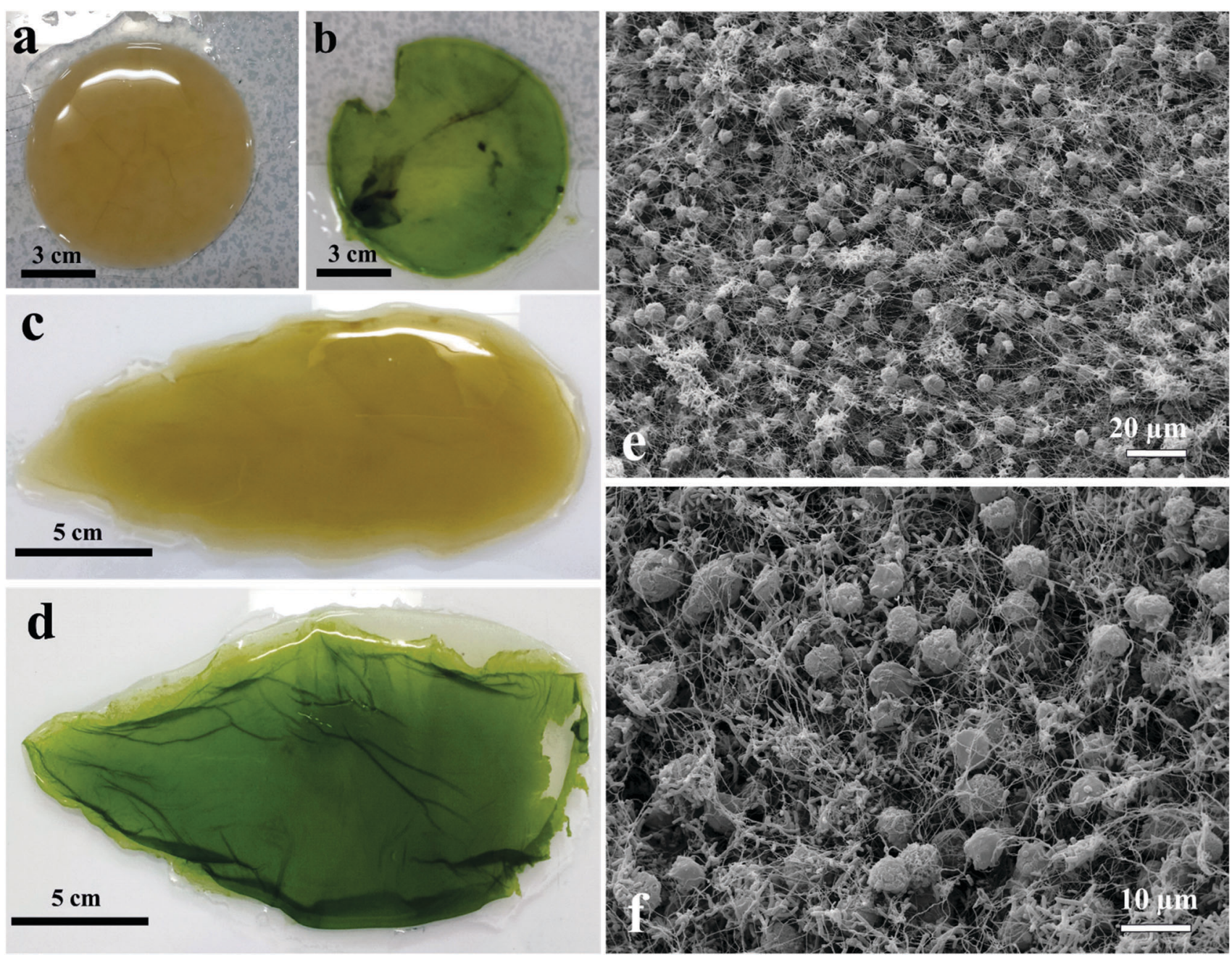

Fig. 8 Optical photographs of different shapes and sizes of cellulose and C. reinhardtii immobilised cellulose (living soft matter) produced by A.aceti (a) flat circularly shaped cellulose gel, (b) microalgae immobilised in a circularly shaped cellulose gel, (c) leaf-shaped bacterial cellulose and (d) leaf-shaped living soft matter with immobilised $C$. reinhardtii into the bacterial cellulose gel. Scanning electron microscopic image of (e) the living soft matter made of cellulose produced from A. aceti and in situ immobilised microalgae C. reinhardtii cultured in a glass beaker and (f) higher magnification image of (e) showing specific areas with the entrapped microalgae in the bacterial cellulose matrix.

Samples from the living biomaterial composites were observed by SEM. The rod-shaped cells (Fig. 7a) and the oval shaped cells with two anterior flagella (Fig. $7 \mathrm{~b}$ ) are A. aceti and $C$. reinhardtii, respectively. When the cells were grown together in a Petri dish, the morphology of the produced biocomposite was different from the one produced in a beaker as shown in Fig. 7e, f and $8 \mathrm{e}, \mathrm{f}$. The number of celluloseproducing bacteria and microalgae cells are both much higher in the case of the biocomposites made in the beaker compared to those made in a Petri dish as evident from Fig. $7 \mathrm{f}$ and $8 \mathrm{f}$. Note that the cellulose fibres are finer in the case of the thin biomaterial made in the Petri dish compared to the thick one in the beaker.

\section{Moulding of the living biomaterial}

We have grown living biomaterial samples of different shapes like spherical bead, cylindrical and leaf-shaped. The shapes like flat circular thick cellulose as shown in Fig. $8 \mathrm{a}$ and $\mathrm{b}$ were made in a beaker while the leaf-shaped living biomaterial was produced in a culture vessel with the shape of a leaf as shown in Fig. 8c and $\mathrm{d}$ with the latter with immobilised microalgae in all cases. It was also observed that the cellulose sheet produced always takes the shape of the culture vessel, hence this property was used in order to "mold" the produced living soft matter into various shapes, sizes and thickness as shown in Fig. 8 and 11.

We produced living soft matter in the shape of a spherical bead as shown in Fig. 11a and b where the latter was with immobilised $C$. reinhardtii cells grown during the cellulose bead formation. This was previously demonstrated by $\mathrm{Hu}$ et $a l^{12}$ for the immobilisation of human osteoblast cells in sphere-like cellulose particles through the interactions between Gluconacetobacter xylinus and the mammalian cells. The SEM image analysis of the living soft matter bead presented in Fig. 11d shows similar morphological characteristics to the living biomaterial produced in the beaker - see Fig. 8f. In the ESI, $\dagger$ 

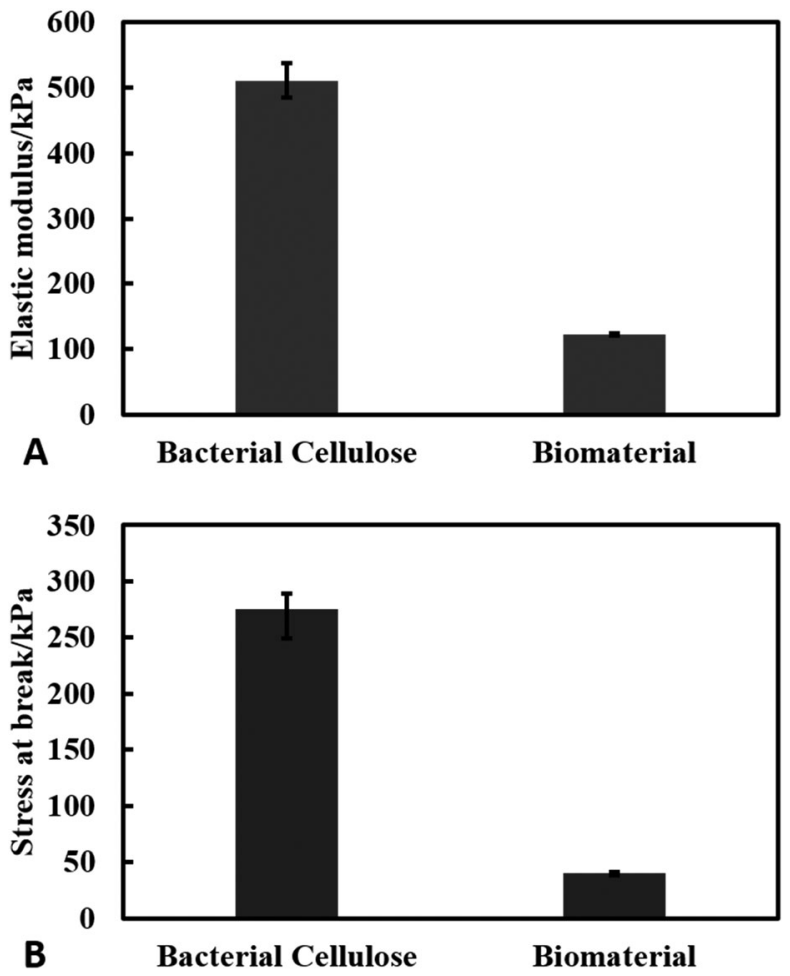

Fig. 9 Results of tensile test of bacterial cellulose and the biomaterial. (A) Young's modulus ( $\mathrm{kPa}$ ) and (B) stress at break ( $\mathrm{kPa}$ ). The experiments were conducted in triplets.

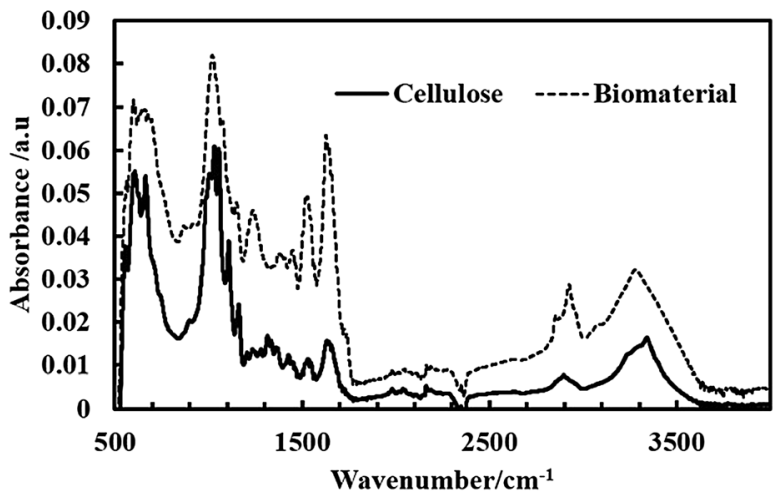

Fig. 10 ATR FT-IR spectra of a film of bacterial cellulose produced by $A$. aceti and living biomaterial produced by the symbiotic growth of $A$. aceti and $C$. reinhardtii.

we also present different strategies for the integration of microalgae into a pre-fabricated slurry of bacterial cellulose gel beads with $A$. aceti.

\section{Mechanical properties of the living biomaterial}

We measured the elastic properties of the material using tensile strength equipment. The elastic modulus of the produced cellulose and the living biomaterial was found to be $510 \pm 30 \mathrm{kPa}$ and $120 \pm 2 \mathrm{kPa}$ respectively as shown in Fig. 9(A). The ultimate tensile strength of the materials was found to be $280 \pm 10 \mathrm{kPa}$ for the bacterial cellulose gel and $40 \pm 2 \mathrm{kPa}$ for the living
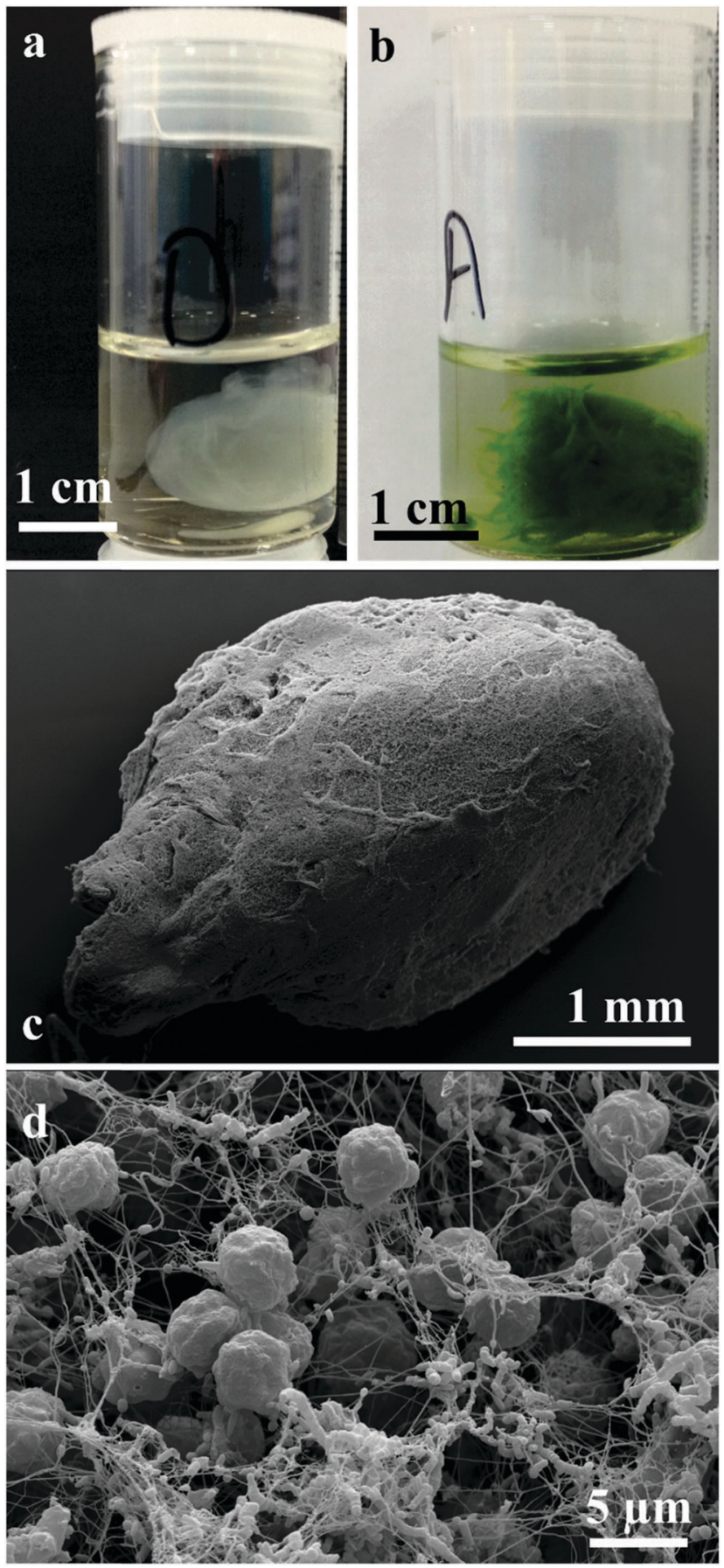

Fig. 11 Photographs of cellulose and C. reinhardtii immobilised cellulose (living biomaterial) produced by $A$. aceti (a) spherical bead of bacterial cellulose grown by $A$. Aceti and (b) microalgae immobilised cellulose bead. (c) Scanning electron micrograph of (a) cellulose bead produced by A. aceti with immobilised C. reinhardtii cells; (d) higher magnification image of the bead showing the microalgae cells enveloped in a mesh of cellulose fibre along with $A$. aceti.

biomaterial shown in Fig. 9(B). The calculations were based on the stress-strain graph shown in Fig. S10 (see ESI $\dagger$ ). The bacterial cellulose gel material produced is much stronger and elastic in nature compared to the living biomaterial. Despite the lower mechanical strength compared with the original 
matrix the living biomaterial is strong enough to be handled and shaped in a variety of molds.

\section{Characterisation of the biomaterial composition}

The carbon and hydrogen contents of $45.30 \pm 0.28$ and $5.22 \pm$ $0.26 \%$, respectively, were found in dried samples of the symbiotic living biomaterial. These results were similar to the reported values of pure cellulose composition of carbon and hydrogen contents of 44.05 and $6.28 \%$, respectively. ${ }^{21}$ Some nitrogen was also detected in the cellulose samples due to the presence of amino acids from the residual bacteria after purification.

The FT-IR spectra of the produced bacterial cellulose as well as the living biomaterial are shown in Fig. 10. The samples obtained in the form of cellulose from A. aceti and the living biomaterial show similar bands to higher absorbance values in the case of the latter. The spectrum in Fig. 10 also shows the similar typical bands reported previously for cellulose based materials. ${ }^{22}$ The spectrum was also used to determine the ratio of allomorphs $\mathrm{I} \alpha$ and $\mathrm{I} \beta .^{23}$ The $\mathrm{I} \alpha / \mathrm{I} \beta$ ratio was found to be 0.82 and 0.84 for cellulose and the living biomaterial respectively which is similar to that reported for bacterial cellulose. ${ }^{24}$ This also shows that the cellulose produced by $A$. acet $i$ is rich in the I $\alpha$ polymorph.

The SEM-EDX analysis was conducted on the cellulose produced by A. aceti and also on the living biomaterials in order to determine the composition shown in Fig. S11 and S12 (ESI $\dagger$ ). The results show higher percentages of carbon and oxygen as expected which correlated with our findings obtained using the CHN analyser. The presence of other elements like osmium, phosphorus, gold and palladium was observed due to sample preparation and coatings before SEM imaging and EDX analysis.

\section{Conclusions}

We have mimicked the relationship between microalgae and fungi, which forms lichens in Nature to produce a novel type of living soft matter based on the symbiosis of other unicellular microorganisms. It was found that A. aceti (NCIMB 8132), which can produce cellulose gels gets into a symbiotic relationship with the photosynthetic $C$. reinhardtii cells which produce oxygen. The microalgae cells were successfully integrated with the $A$. aceti cells in a symbiotic relationship by varying the concentration ratios of the initial culture media (TAP and GY media). It was found that at the ratio containing $70: 30 \mathrm{GY}$ : TAP media $(\mathrm{v} / \mathrm{v})$ both the microorganisms grow efficiently and integrate together within the produced bacterial cellulose gels. The immobilisation of the microalgae was done in real time along with the bacterial cellulose production. Since the microalgae $C$. reinhardtii have the ability to produce hydrogen under nutrient deprived conditions, such living soft matter may have advantages like improved hydrogen production ${ }^{13}$ and various other metabolite extractions. In the past, microalgae $C$. reinhardtii (cc124 strain) were immobilised in the alginate gel in order to increase their hydrogen production efficiency compared to suspension culture. ${ }^{13}$ The developed microalgae immobilisation procedure can be used to produce natural microalgae biofilms to be potentially integrated in the artificial leaf devices. ${ }^{13}$ The described approach of combining unicellular microorganisms working in symbiosis by passing metabolites to each other can lead to the development of other symbiotic pairs, where one type of cells builds the extracellular matrix in which the other type of cells integrates and provides essential metabolites. This technique may find applications in the development of a range of biological tissues and living soft matter systems and could be possibly extended to more than two symbiotic microorganisms, which might allow the creation of multifunctional, living and self-replicating systems.

\section{Acknowledgements}

The authors are grateful to Mr Tony Sinclair for the SEM images and to Dr Tommy Horozov and Mr Khaled Althubeiti (University of Hull) who helped us with the tensile strength experiments. V. N. P acknowledges financial support from the EU COST Action CM1101.

\section{Notes and references}

1 S. D. Leavitt, E. Kraichak, M. P. Nelsen, S. Altermann, P. K. Divakar, D. Alors, T. L. Esslinger, A. Crespo and T. Lumbsch, Mol. Ecol., 2015, 24, 3779-3797.

2 V. Ahmadjian, The lichen symbiosis, John Wiley and Sons Ltd, Chichester, England, 1993.

3 R. Honegger, Lichenologist, 1998, 30, 193-212.

4 R. Honegger, New Phytol., 1993, 125, 659-677.

5 M. Grube, M. Cardinale, J. V. de Castro, H. Muller and G. Berg, ISME J., 2009, 3, 1105-1115.

6 B. P. Hodkinson and F. Lutzoni, Symbiosis, 2009, 49, 163-180.

7 T. D. Brock, M. T. Madigan, J. M. Martinko and J. Parker, Biology of microorganisms, Prentice-Hall, London, England, UK, 7th edn, 1994.

8 S. Jasti, M. E. Sieracki, N. J. Poulton, M. W. Giewat and J. N. Rooney-Varga, Appl. Environ. Microbiol., 2005, 71, 3483-3494.

9 Z. Guo and Y. Tong, J. Appl. Phycol., 2014, 26, 1483-1492.

10 N. M. N. Ton and V. V. M. Le, Int. Food Res. J., 2011, 18, 983.

11 A. Rezaee, H. Godini and H. Bakhtou, Environ. Eng. Manage. J., 2008, 7, 589-594.

12 Y. Hu, J. M. Catchmark and E. A. Vogler, Biomacromolecules, 2013, 14, 3444-3452.

13 A. A. K. Das, M. M. N. Esfahani, O. D. Velev, N. Pamme and V. N. Paunov, J. Mater. Chem. A, 2015, 3, 20698-20707.

14 P. Raspor and D. Goranovic, Crit. Rev. Biotechnol., 2008, 28, 101-124.

15 R. A. Andersen, Algal culturing techniques, Elsevier/Academic Press, Burlington, Mass., 2005.

16 J. D. Fontana, A. M. De Souza, C. K. Fontana, I. L. Torriani, J. C. Moreschi, B. J. Gallotti, S. J. De Souza, G. P. Narcisco, 
J. A. Bichara and L. F. X. Farah, Appl. Biochem. Biotechnol., 1990, 24-25, 253-264.

17 T. H. Chrzanowski, R. D. Crotty, J. G. Hubbard and R. P. Welch, Microb. Ecol., 1984, 10, 179-185.

18 P. Breeuwer, J. Drocourt, F. M. Rombouts and T. Abee, Applied and Environmental Microbiology, 1996, 62, 178-183.

19 P. Breeuwer, J. L. Drocourt, N. Bunschoten, M. H. Zwietering, F. M. Rombouts and T. Abee, Appl. Environ. Microbiol., 1995, 61, 1614-1619.
20 L. L. Beer, E. S. Boyd, J. W. Peters and M. C. Posewitz, Curr. Opin. Biotechnol., 2009, 20, 264-271.

21 D. Klemm, D. Schumann, U. Udhardt and S. Marsch, Prog. Polym. Sci., 2001, 26, 1561-1603.

22 H. G. Shirk and G. A. Greathouse, Anal. Chem., 1952, 24, 1774-1775.

23 T. Imai and J. Sugiyama, Macromolecules, 1998, 31, 6275-6279.

24 C. Castro, R. Zuluaga, C. Álvarez, J.-L. Putaux, G. Caro, O. J. Rojas, I. Mondragon and P. Gañán, Carbohydr. Polym., 2012, 89, 1033-1037. 